\title{
Determination of Inter-leaf Translocated Free Glyphosate in Arabidopsis thaliana using Liquid Chromatography Tandem Mass Spectrometry (LC- MS/MS) after Derivatization with Fluorenylmethyloxycarbonyl Chloride (FMOC-Cl)
}

\author{
Misjudeen Raji ${ }^{1 *}$, Altanbadralt Sharkhuu², Ma'an Amad, Jasmeen Merzaban² and Christoph Gehring ${ }^{2}$ \\ ${ }^{1}$ Analytical Core Lab, King Abdullah University of Science and Technology, Thuwal, Saudi Arabia \\ ${ }^{2}$ Biological, Environmental Science and Engineering, King Abdullah University of Science and Technology, Thuwal, Saudi Arabia
}

\begin{abstract}
Glyphosate is a broad-spectrum herbicide widely used for eliminating weeds in crop fields. Its mode of action is believed to be via translocation from the source to the sink tissues where it then interferes with the activities of 5-enol-pyruvylshikimate-3-phosphate synthase (EPSPS). In this study, the translocation of glyphosate in the leaves of Arabidopsis thaliana was investigated using an HPLC-MS/MS method following derivatization of the secondary amino group in the analyte using $\mathrm{N}$-(9-fluorenylmethoxycarbonyloxy) chloride. To eliminate the errant precipitation that occurred when the reagent and the analyte are mixed, optimization of this method was required. The method linearity has a correlation coefficient higher than 0.99 over the concentration range of $0.005-2 \mu \mathrm{M}$. The limits of detection and quantitation were estimated to be $0.002 \mu \mathrm{M}$ and $0.008 \mu \mathrm{M}$ respectively. The repeatability of the method (as $\%$ R.S.D) ranged from $10 \%$ to $13 \%$. The presented method was employed for the determination of free glyphosate in young untreated leaves of the specimen plants after treating a single leaf and allowing it to stand for 12 hours.
\end{abstract}

Keywords: Glyphosate; FMOC-Cl; Electrospray; Mass spectrometry; Translocation; Arabidopsis thaliana

\section{Introduction}

Glyphosate [N-(phosphonomethyl) glycine] is a non-selective herbicide that has gained considerable interest in recent years. It is widely used in fields of cereals crops to eliminate weeds while preserving the crops of interest, which are usually genetically modified to resist glyphosate action. The wide spread use of glyphosate has raised environmental concerns as to the fate of this compound when washed into ground water [1]. These concerns have necessitated the development of analytical methods capable of detecting this compound in various matrices such as soil [2] and environmental water samples [3], fruits and vegetables [4,5], as well as in human serum [6]. Aside the interest in measuring glyphosate in these different matrices, there is significant evidence that the efficacy of the herbicide is determined by its extent of translocation from a source location to other tissues of a plant [7], highlighting the interest in being able to conveniently measure the amount of glyphosate translocated to different tissues.

In general, the most widely used methods for analyzing glyphosate in various matrices and sample types are liquid chromatography coupled to UV-VIS detection, fluorescence detection, or electrospray tandem mass spectrometry [8-11], ion chromatography coupled with different detection methods such as conductivity and mass spectrometry $[12,13]$, and Gas Chromatography coupled with Mass Spectrometry (GC-MS) $[14,15]$ or with Flame Photometric Detection (GC-FPD) [16]. Majority of these methods are not direct and also involve many challenges. The major challenge in the analysis of glyphosate stems from the chemical nature of the compound. The absence of a chromophoric or fluorophoric moiety on the molecule, for example, limits the application of spectrophotometric techniques such as UVVIS absorption and fluorescence detection for direct measurement. In addition, chromatographic separation of the molecule poses a difficulty due to low retainability on commonly used column packing materials in reverse phase liquid chromatography. Its high polarity also makes it unamenable for direct measurement via gas chromatography. The most common method of circumventing these problems is by derivatizing the compound, either to impart a chromogenic or fluorogenic character, thereby improving its spectrophotometric detection, or to make it more volatile for GC analysis. The derivatization step may also lead to decreased polarity of glyphosate, and by so doing improve its retention in reverse phase liquid chromatography. Among the most frequently utilized derivatization reagents for analyzing glyphosate in reverse phase liquid chromatography analysis is 9-fluorenylmethylchloroformate (FMOC-Cl) [17]. The FMOC moiety imparts some degree of non-polarity to the molecule while also making it amenable to UV detection.

To date, the most common method for determining glyphosate translocation in plants involves the use of ${ }^{14} \mathrm{C}$-glyphosate as part of the treatment solution followed by scintillation counting. As this radioactive analog is being phased out by many manufacturers, it is pertinent to explore alternative methods for monitoring glyphosate translocation in plant tissues. In this study, we explore the possibility of determining free translocated glyphosate in plants using the FMOC$\mathrm{Cl}$ derivatization technique coupled with LC-ESI-MS/MS detection.

${ }^{*}$ Corresponding author: Misjudeen Raji, Analytical Core Lab, King Abdullah University of Science and Technology, Thuwal, 3327, Building 3, Level 3 4700 KAUST, Thuwal, 23955, Saudi Arabia, Tel: +966-544-700-353; E-mail: Misjudeen.raji@kaust.edu.sa

Received December 19, 2013; Accepted January 31, 2014; Published February 03, 2014

Citation: Raji M, Sharkhuu A, Amad M, Merzaban J, Gehring C (2014) Determination of Inter-leaf Translocated Free Glyphosate in Arabidopsis thaliana using Liquid Chromatography Tandem Mass Spectrometry (LC-MS/MS) afte Derivatization with Fluorenylmethyloxycarbonyl Chloride (FMOC-Cl). J Anal Bioanal Tech S2: 007. doi:10.4172/2155-9872.S2-007

Copyright: (C) 2014 Raji M, et al. This is an open-access article distributed unde the terms of the Creative Commons Attribution License, which permits unrestricted use, distribution, and reproduction in any medium, provided the original author and source are credited. 
Citation: Raji M, Sharkhuu A, Amad M, Merzaban J, Gehring C (2014) Determination of Inter-leaf Translocated Free Glyphosate in Arabidopsis thaliana using Liquid Chromatography Tandem Mass Spectrometry (LC-MS/MS) after Derivatization with Fluorenylmethyloxycarbonyl Chloride (FMOC-Cl). J Anal Bioanal Tech S2: 007. doi:10.4172/2155-9872.S2-007

Page 2 of 6

The work focused on measuring translocated glyphosate in younger untreated leaves after applying glyphosate to older leaves of the same plant. The objective is to establish a proof of concept based on LCESI-MS/MS technique as a viable alternative method for measuring glyphosate translocation. Arabidopsis thaliana was used as a model plant in this study.

\section{Experimental}

\section{Materials}

Glyphosate (>97\% purity), Fluorenylmethyloxycarbonyl chloride (FMOC-Cl), and sodium tetraborate were purchased from SigmaAldrich (St. Louis, MO, USA). LC-MS grade methanol was purchased from Fisher Scientific (Pittsburgh, PA, USA). Ammonium carbonate, ammonium hydroxide, and formic acid were purchased from Fisher Scientific (Pittsburgh, PA, USA). Water was prepared using a Millipore Milli-Q purification system (Bedford, MA, USA).

\section{Calibration standards}

A $50 \mathrm{mM}$ glyphosate standard stock solution was prepared using HPLC grade water. Two sets of standard calibration solutions were prepared from the stock by diluting accurately measured amounts in either $0.25 \mathrm{~N} \mathrm{HCl}$ prepared in LC-MS grade water or $0.25 \mathrm{~N} \mathrm{HCl}$ extract of carefully abscised and weighed pristine Arabidopsis leaves (with plant matrix concentration at $40 \mathrm{mg}$ tissue per $\mathrm{mL}$ of solution). The tested concentration range was from $0.001 \mu \mathrm{M}$ to $2 \mu \mathrm{M}$.

\section{Glyphosate treatment and extraction of plant samples}

Selected leaves of several three-weeks-old Arabidopsis plants grown under $80 \mathrm{mM} \mathrm{s}^{-1} \mathrm{~m}^{-2}, 16 \mathrm{~h}$ light conditions were treated at onset of light time with $6 \mu \mathrm{L}$ of $200 \mathrm{mM}$ glyphosate and kept under the same growth condition. After 12 hours, three youngest leaves from each plant were carefully abscised and weighed into a clean $1.8 \mathrm{~mL}$ Eppendorf tube and stored in $-20^{\circ} \mathrm{C}$ until extraction. Two methods were evaluated for the extraction. The first method involved grinding abscised leaves under liquid nitrogen and extracting with a water/methanol mixture, while simple immersion in $0.25 \mathrm{~N} \mathrm{HCl}$ was used in the second method. The grinding method was found to be cumbersome and led to loss of samples. The method of immersion was therefore selected for the extraction. In this method, frozen leaf samples were thawed at $60^{\circ} \mathrm{C}$ for 15 minutes followed by addition of $0.25 \mathrm{~N} \mathrm{HCl}$ and then incubation at $60^{\circ} \mathrm{C}$ for additional 15 minutes. The volumes of extraction solvent were normalized for each sample based on the leaf weights to maintain similar matrix concentration of $40 \mathrm{mg} / \mathrm{ml}$ across all samples. This facilitated the normalization of glyphosate concentration to the weight of the leaf tissues. Three biological replicates were used for each plant. Positive controls were prepared in plant extract using the same amount of glyphosate. All standard and sample solutions were stored in clean $1.8 \mathrm{~mL}$ Eppendorf tubes until analysis.

\section{Derivatization with FMOC-Cl}

Both the calibration standards and the extracted samples were derivatized using the FMOC-Cl method with minor modifications. Briefly, $50 \mu \mathrm{L}$ of each standard and sample solution was first neutralized by adding $50 \mu \mathrm{L}$ of $1 \mathrm{M}$ ammonium hydroxide in $1.8 \mathrm{~mL}$ Eppendorf tubes. After vortexing for 15 seconds, $850 \mu \mathrm{L}$ of the derivatization buffer mixture (prepared by mixing methanol with a $5 \%$ sodium tetraborate solution in 30/70 ratio, $\mathrm{pH}$ was adjusted to $\sim 9.1$ using 1 $\mathrm{M}$ ammonium hydroxide) was added to the solution and vortexed gently. Subsequently, $50 \mu \mathrm{L}$ of a $10 \mathrm{mg} / \mathrm{ml}$ FMOC solution was added and each tube was vortexed again for 30 seconds and spun at 10,000 rpm for 2 minutes to allow all the solutions in the tube cap to drain down into the tube. Derivatization was allowed to proceed overnight at room temperature. Two control plant samples (grown under the same condition but prevented from coming into contact with glyphosate) were similarly extracted in $0.25 \mathrm{~N} \mathrm{HCl}$ and $50 \mu \mathrm{L}$ of each was derivatized using the same optimized procedure described above. In addition, 2 sets of fortified samples (prepared by spiking known amounts of glyphosate into blank LC-MS grade water and blank plant extracts at two concentration levels) were derivatized using the same optimized derivatization procedure. Derivatized samples were collected into labeled HPLC vials after 16 hours in readiness for LC-MS analysis.

\section{LC-ESI-MS/MS analysis of derivatized samples}

Prior to LC-MS/MS analysis, a direct infusion ESI-MS analysis of pure glyphosate $(5 \mu \mathrm{M})$ derivatized with FMOC was performed on a Thermo Scientific TSQ Vantage triple quadrupole mass spectrometer (Thermo Scientific, San Jose, CA, USA) equipped with a HESI-II electrospray source using a metal spray needle in order to develop a Selected Reaction Monitoring (SRM) method for glyphosate determination. The built-in syringe pump of the instrument was used to carry out the infusion and the flow was mixed with a 50/50 methanol/ water mobile phase from the LC system using a zero dead volume T-union. Syringe pump flow was $30 \mu \mathrm{L} / \mathrm{min}$ and LC flow rate was 300 $\mu \mathrm{L} / \mathrm{min}$. The instrument was tuned for the mass of the derivatized product (391.9) and appropriate parent-daughter fragmentation transitions were determined and optimized. LC-MS/MS analysis was performed by injecting $10 \mu \mathrm{L}$ of each standard and sample onto a C8 column (Agilent Zorbax Eclipse Plus, $4.6 \mathrm{~mm} \times 100 \mathrm{~mm}, 5 \mu \mathrm{m}$ ) and eluting in gradient mode using a binary solvent mix comprising a 2 $\mathrm{mM}$ ammonium carbonate solution in water (mobile phase A) and a 95/5 Methanol/water solution containing $2 \mathrm{mM}$ ammonium carbonate (mobile phase B). In the gradient program, the initial mobile phase composition of $40 \%$ B was held for $0.5 \mathrm{~min}$ and then ramped to $95 \%$ $\mathrm{B}$ over a period of 3 minutes, whereupon it was held for another 1.5 minutes before being returned to the initial composition and then held for another 1 minute for a total gradient run time of 7 minutes. The mobile phase flow rate was $0.6 \mathrm{~mL} / \mathrm{min}$. All analyses were carried out in positive ionization mode with a spray voltage of $3.5 \mathrm{kV}$. The source temperature and the ion transfer tube temperature were $300^{\circ} \mathrm{C}$ and $270^{\circ} \mathrm{C}$. The $\mathrm{m} / \mathrm{z}$ calibration of the mass analyzer was performed in positive ESI ionization mode using a solution containing polytyrosine according to the manufacturer's guidelines. Instrument control and data analysis were performed using the Thermo Scientific Xcalibur software.

\section{Results and Discussion}

The method utilized here involved the use of FMOC-Cl to derivatize
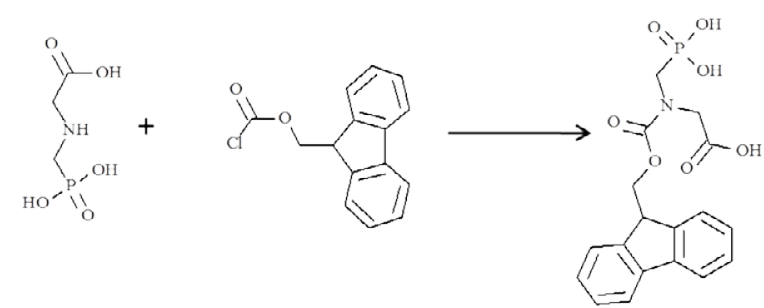

Glyphosate

FMOC-Cl

Scheme 1: Derivatization of Glyphosate using FMOC-Cl. 

thaliana using Liquid Chromatography Tandem Mass Spectrometry (LC-MS/MS) after Derivatization with Fluorenylmethyloxycarbonyl Chloride (FMOC-CI). J Anal Bioanal Tech S2: 007. doi:10.4172/2155-9872.S2-007

the secondary amine moiety on glyphosate (as depicted in the reaction outlined in Scheme 1), followed by LC-MS/MS analyses of the FMOC derivative using positive ion mode ESI-MS. Both derivatization and LC-MS/MS procedures were optimized to achieve optimum detection of the derivatized glyphosate (Scheme 1).

\section{Optimization of derivatization procedure}

Although the FMOC-Cl derivatization technique has been widely utilized in the analysis of glyphosate in different matrices, it is still important to perform an optimization of the procedure in order to ensure reliable results, particularly when considering the investigation of unstudied matrices. Upon applying the FMOC-Cl derivatization technique, we noted that the FMOC reagent slowly precipitates out of the derivatization medium over time (whether glyphosate was in aqueous or plant extract media), invariably affecting its availability for derivatizing the analyte of interest (i.e. glyphosate). As such, one of our prime optimization objectives was to eliminate- or at least minimize-this phenomenon. In order to achieve this, response surface experimental design methodology was used to investigate the effects of different experimental parameters on the derivatization procedure (data and experimental details are not shown here). Another derivatization reagent (FMOC-Succinimide) was also utilized for the derivatization of glyphosate as part of the optimization process. Results showed that derivatization efficiency is enhanced when a molar excess of $>1000 \mathrm{FMOC}-\mathrm{Cl} /$ glyphosate molar ratio is used and derivatization is carried out between 25 and $50^{\circ} \mathrm{C}$ at moderately alkaline $\mathrm{pH}(8-10)$. Also, derivatization time of 60 minutes was found to be sufficient and there was no significant increase in yield for longer durations. In all of these experiments, the extent of precipitation was greatly influenced by the starting concentration of the FMOC reagent as well as the composition of the buffer medium. It was therefore necessary to reach a fine balance between these two parameters. Several buffer compositions were tested, including binary mixtures of methanol and $2 \mathrm{mM}$ ammonium carbonate, methanol and 5\% sodium borate, as well as methanol and $2 \mathrm{mM}$ ammonium bicarbonate (with methanol composition in each binary mixture varying from 20 to 70\%). Of all the studied buffers, the binary mixture of methanol and $5 \%$ sodium borate comprising $70 \%$ methanol and $30 \%$ sodium borate was found to produce the least amount of precipitation. This buffer mixture also gave higher signal intensity compared to the other two (even though the methanol $/ 2 \mathrm{mM}$ ammonium carbonate mixture produced slightly better chromatographic peak shape). The 70/30 methanol/borate buffer system was thus selected as the derivatization medium for all standards and samples. The use of FMOC-Succinimide did not have any effect on the precipitation during derivatization. However, the yield was significantly lower (about 1 order of magnitude) compared to FMOC$\mathrm{Cl}$ derivatization. The reason behind this difference in yield between the two FMOC reagents needs further investigation.

\section{LC-MS/MS analysis}

The MS source parameters were optimized for enhanced detection of the FMOC-glyphosate derivative by tuning the instrument using the analyte $\mathrm{m} / z$ of 391.9 while infusing a $10 \mu \mathrm{M}$ derivatized glyphosate solution into an LC pump flow of 50/50 methanol/water. Once stable and appreciable signal intensity was achieved for the parent mass,

\begin{tabular}{|c|c|c|c|}
\hline Precursor Ion & S-Lens & Product Ion & Collision Energy \\
\hline 391.9 & 74 & 88.2 & 18 \\
\hline & & 178.2 & 53 \\
\hline & & 179.2 & 27 \\
\hline
\end{tabular}

Table 1: Fragment ions used for SRM studies and their respective energies.

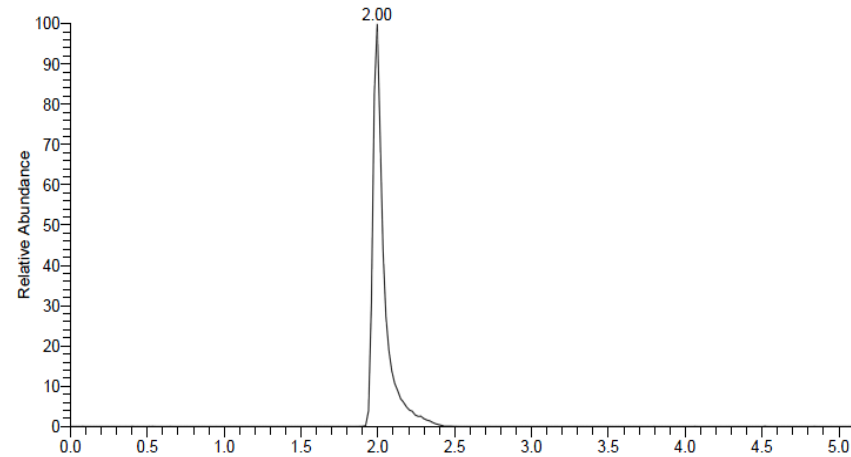

Figure 1: Total ion chromatogram of ion 88.2 obtained for a derivatized $10 \mu \mathrm{M}$ solution of pure glyphosate.

collision induced fragmentation was performed and fragmentation parameters (such as collision gas pressure and collision energy) were optimized. The three most abundant fragment ions obtained under optimized fragmentation conditions and their respective energies are listed in Table 1. In order to establish the most appropriate fragment ion for quantitation, the structures as well as the fragmentation pathways for these three fragment ions were critically examined. Based on the structures of the ions in relation to the precursor ion, fragment ion 88.2 appears to be more specific to glyphosate while ions 178.2 and 179.2 could have resulted from any secondary-amine-bearing molecule derivatized by FMOC. As such, ion 88.2 was selected as the quantifying ion in this study.

For optimum chromatographic separation, a $95 / 5$ methanol/2 mM ammonium carbonate mixture was selected as the organic phase in the gradient elution in conjunction with a $2 \mathrm{mM}$ ammonium carbonate aqueous phase after assessing several other mobile phase compositions. This mobile phase composition was selected based on the better peak shape if afforded compared to the other mobile phases investigated. Under these optimized conditions, the FMOC-glyphosate derivative eluted from the column at about 2.00 minutes. A typical chromatogram obtained for derivatized sample of a $10 \mu \mathrm{M}$ solution of pure glyphosate is presented in Figure 1.

\section{Method Figures of Merit}

The method linearity was assessed using the two sets of calibration data obtained from FMOC-derivatized glyphosate spiked in 0.25 $\mathrm{N} \mathrm{HCl}$ and $40 \mathrm{mg} / \mathrm{ml}$ leaf extract in $0.25 \mathrm{~N} \mathrm{HCl}$. The investigated concentration range for both calibration sets is $0.005 \mu \mathrm{M}-2 \mu \mathrm{M}$. A linear regression fit was used for both calibration curves with $\mathrm{R} 2$ values in both cases $>0.99$ over the range of concentration (Figure $2 a$ and $2 b$ ). Precision (expressed as \% relative standard deviation) was determined by analyzing 3 glyphosate-spiked samples after FMOC derivatization. The spiking levels were $0.05,0.1$ and $0.2 \mu \mathrm{M}$. The blank extract used for these spiking studies was tested to ensure that it does not contain glyphosate. The RSD ranged from $10-13 \%$. The recoveries for the same spiked samples ranged from $67 \%$ to $114 \%$. The Limits of Detection (LOD) and Quantitation (LOQ) were estimated as 0.002 and $0.008 \mu \mathrm{M}$, respectively. The effect of matrix composition on the analyte response was also examined across the linear range. The results show that there is approximately $50 \%$ reduction in analyte signal on average due to the matrix component effect. This further accentuates the importance of taking matrix effects into proper consideration when quantifying glyphosate in food and plant matrices, where the effect is generally more pronounced. 
Citation: Raji M, Sharkhuu A, Amad M, Merzaban J, Gehring C (2014) Determination of Inter-leaf Translocated Free Glyphosate in Arabidopsis thaliana using Liquid Chromatography Tandem Mass Spectrometry (LC-MS/MS) after Derivatization with Fluorenylmethyloxycarbonyl Chloride (FMOC-Cl). J Anal Bioanal Tech S2: 007. doi:10.4172/2155-9872.S2-007

Page 4 of 6

\section{Analysis of glyphosate in arabidopsis leaves}

Analysis of glyphosate translocation in plants using the ${ }^{14} \mathrm{C}$ analog of the herbicide has been reported in the literature [18,19]. Given the diminishing production of this radio-labeled analog, it is conceivable that this method will soon become inapplicable. As such, an alternative method for tracking glyphosate translocation needs to be explored. The main objective of this study was to evaluate the LC-MS/MS method for determining translocated glyphosate into untreated Arabidopsis leaves (i.e. the youngest leaves). To this end, the oldest leaf of each of seven 3-weeks old Arabidopsis plants was treated with $6 \mu \mathrm{L}$ of $200 \mu \mathrm{M}$ glyphosate. Three youngest leaves of each plant were carefully incised

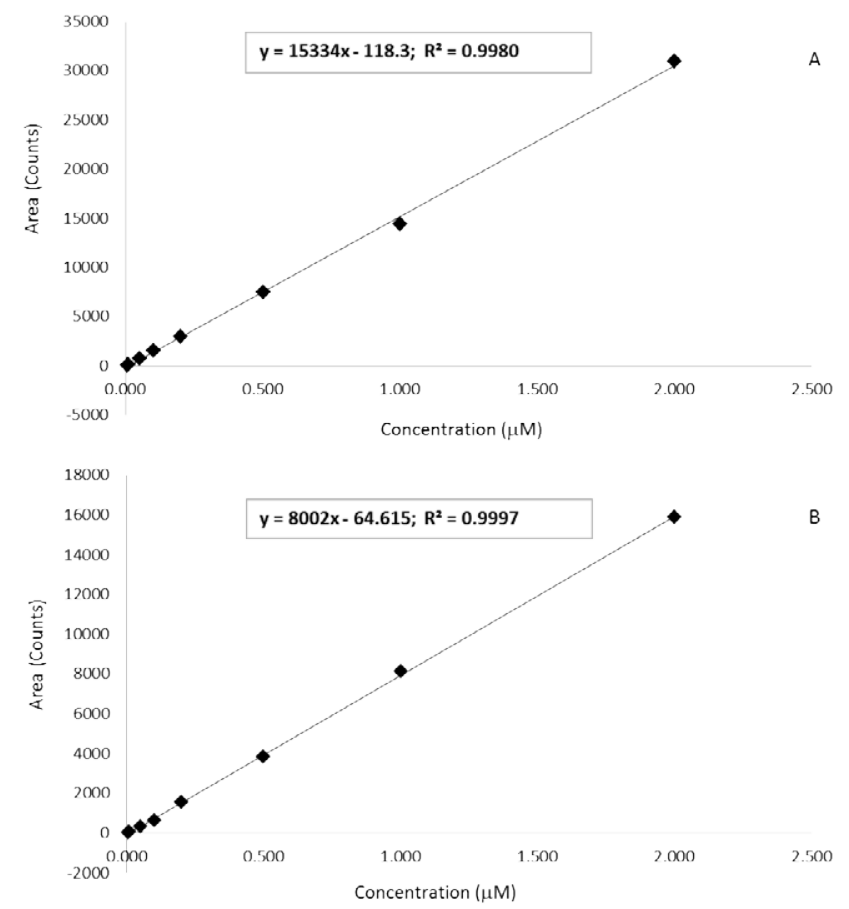

Figure 2: Calibration curve for glyphosate in $\mathrm{A}$ ) $0.25 \mathrm{~N} \mathrm{HCl}$, and B) leaf extract of Arabidopsis in $0.25 \mathrm{~N} \mathrm{HCl}$. after 12 hours, extracted with $0.25 \mathrm{~N} \mathrm{HCl}$, derivatized, and analyzed for glyphosate using the method described above. The established retention window of glyphosate in both pure standards as well as matrix-matched standards, coupled with the qualifying SRM ions, was used to confirm the presence of glyphosate in the tested leaves. The two calibration curves (based on pure standards in solvent and in clean extract) were used to quantify glyphosate. The detected amount of glyphosate in the untreated leaves ranged from 0.007 to $0.04 \mu \mathrm{M}$ (corresponding to 6.04 $-34.4 \mu \mathrm{g} / \mathrm{mg}$ leaf tissue when normalized to extracted tissue weight). A comparison of the chromatogram of three pooled young untreated leaves to that of a single older leaf treated with glyphosate is presented in Figure 3. Analysis of the negative controls, obtained by harvesting three young leaves of untreated plants grown under similar conditions and for the same duration, presented no glyphosate (Figure 4).

The treated leaves for each set of tested untreated leaves were also analyzed for glyphosate. The detected concentrations in treated leaves were less than the measured concentration of the positive control spiked at the same concentration of glyphosate into clean leaf extract by up to $95 \%$ (Figure 5). This provides further evidence, in addition to the detection in untreated leaves, that some amount of glyphosate was lost from the treated leaves. Moreover, summing the detected amounts in the treated and the corresponding untreated leaves did not amount to the expected concentration when compared to the positive control. One logical explanation of this is that some glyphosate may irreversibly bind the penultimate enzyme of shikimate pathway, EPSPS (5-Enolpyruvylshikimate 3-Phosphate Synthase [20,21] with such a high affinity that it becomes irrecoverable. It will therefore require a more rigorous extraction method to release any enzyme-bound glyphosate. For the scope of this study, the simple extraction method reported above was deemed sufficient.

\section{Conclusion}

We have applied a simple method coupling optimized FMOCderivatization with LC-MS/MS for the determination of free glyphosate in both treated and untreated leaves of Arabidopsis. The results show that some portion of the glyphosate applied to older leaves of the plant translocated to younger leaves over time. These results show that the LC-MS/MS technique is a potential candidate method for measuring glyphosate translocation. To the best of our knowledge, there has
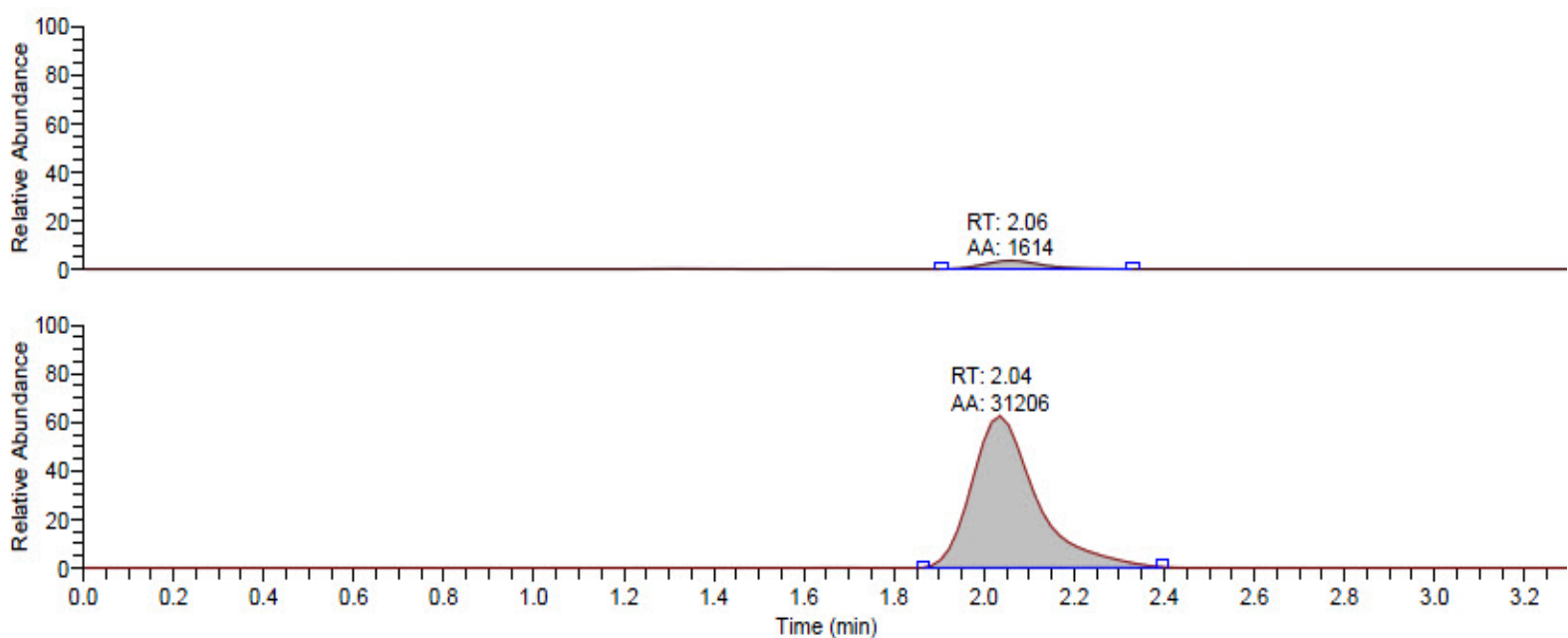

Figure 3: Stacked total ion chromatograms of three young untreated leaves (pooled) (top) and a single older leaf treated with $6 \mu \mathrm{L}$ of $200 \mathrm{mM}$ glyphosate (bottom) after 12 hours of treatment (both plots are normalized to same scale). 
Citation: Raji M, Sharkhuu A, Amad M, Merzaban J, Gehring C (2014) Determination of Inter-leaf Translocated Free Glyphosate in Arabidopsis thaliana using Liquid Chromatography Tandem Mass Spectrometry (LC-MS/MS) after Derivatization with Fluorenylmethyloxycarbonyl Chloride (FMOC-Cl). J Anal Bioanal Tech S2: 007. doi:10.4172/2155-9872.S2-007
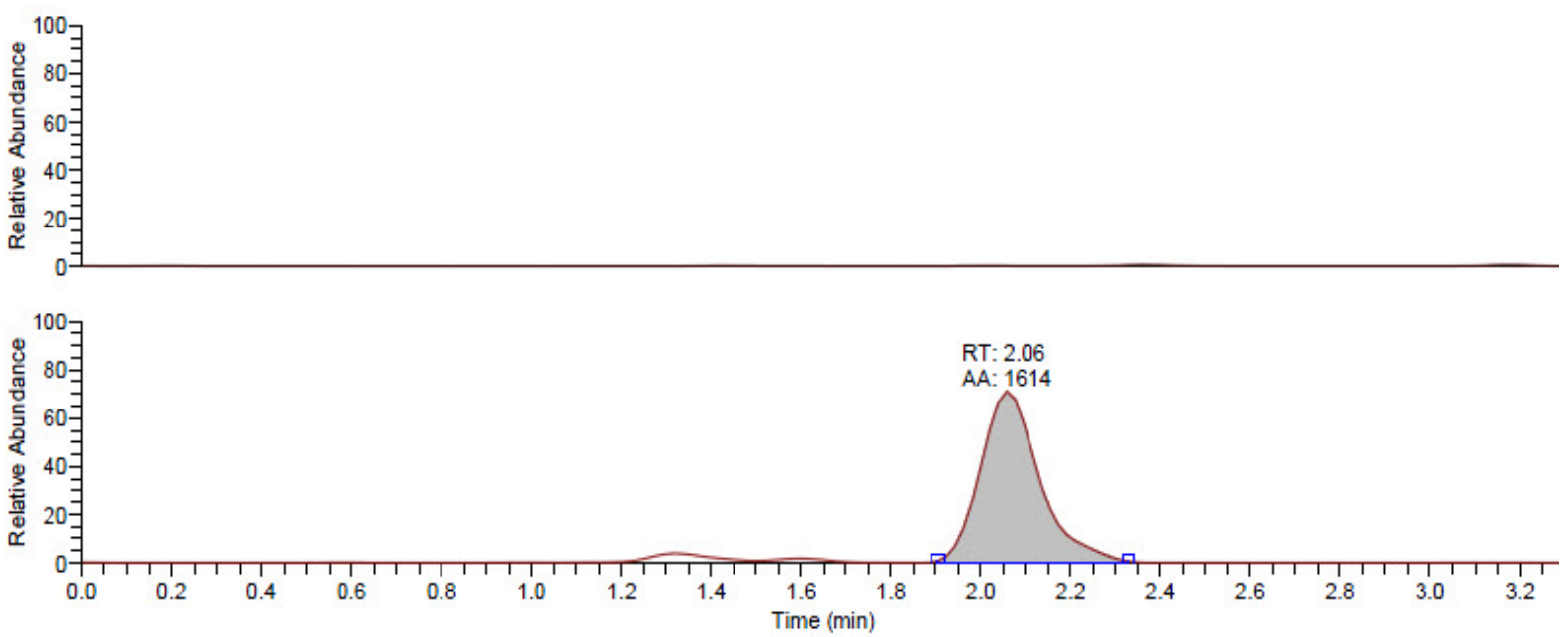

Figure 4: Stacked total ion chromatograms of pristine leaf extract (top) used as a negative control and three pooled young untreated leaves after 12 hours of treating an older leaf of the same plant with glyphosate (bottom) (both plots are normalized to same scale).

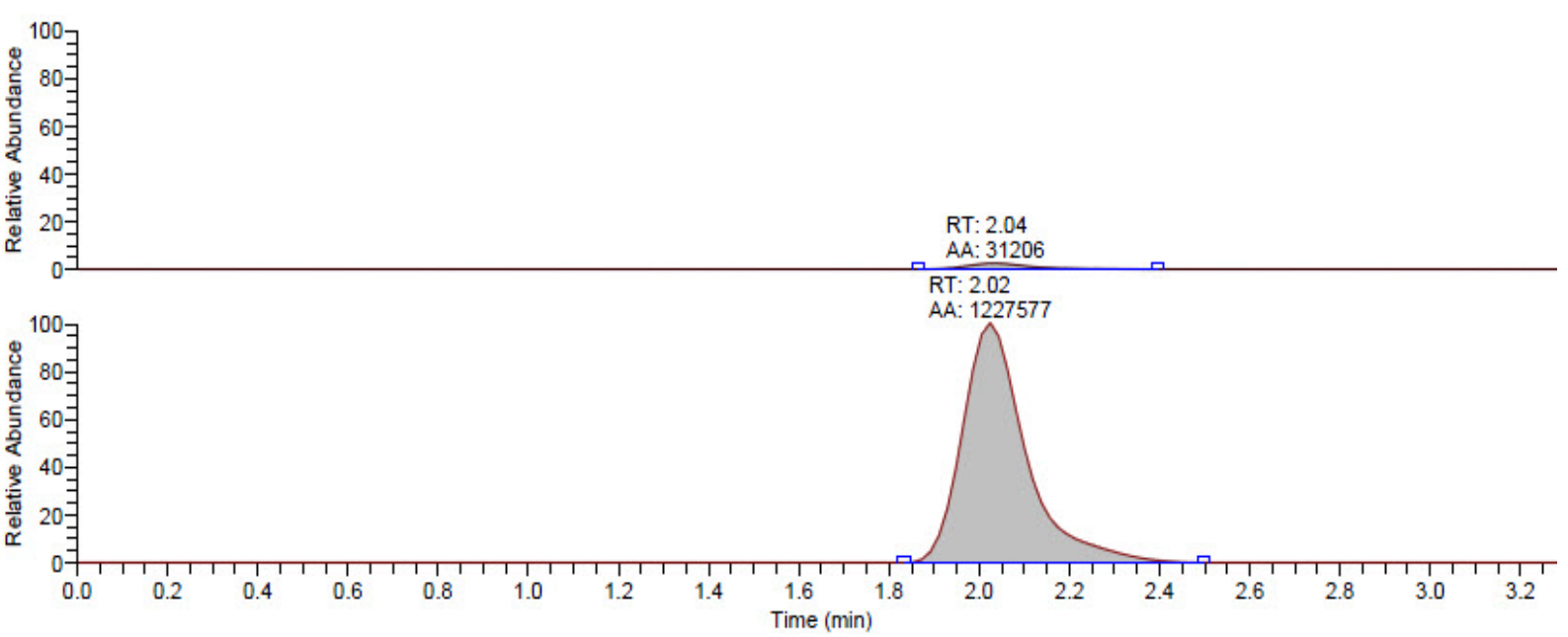

Figure 5: Overlay of the total ion chromatograms of a whole leaf treated with $6 \mu \mathrm{L}$ of $200 \mathrm{mM}$ glyphosate after 12 hours of treatment (top) and a solution of pristine leaf extract spiked with glyphosate to the same final concentration - used as positive control (bottom) (both plots are normalized to same scale).

not been an earlier account of such targeted detection of inter-leaf translocation of glyphosate or similar herbicides in plants using this technique. A more detailed study that couples this technique with the physiological factors influencing the phenomenon of translocation is necessary in order to better evaluate the efficacy but this is outside the scope of the present study. It was also shown that matrix effects can play a significant role in the determination of translocated glyphosate in leaf tissue by this method as evidenced by the $50 \%$ reduction in signals between solvent-spiked and matrix-spiked glyphosate solutions. It is therefore recommended to regularly use matrix-matched calibration standards for quantification.

\section{References}

1. Torstensson L, Borjesson E, Stenström J (2005) Efficacy and fate of glyphosate on Swedish railway embankments. Pest Manag Sci 61: 881-886.

2. Colombo SM, Masini JC (2011) Developing a fluorimetric sequential injection methodology to study adsorption/desorption of glyphosate on soil and sediment samples. Microchemical Journal 98: 260-266.

3. Guo ZX, Cai Q, Yang Z (2005) Determination of glyphosate and phosphate in water by ion chromatography--inductively coupled plasma mass spectrometry detection. J Chromatogr A 1100: 160-167.

4. Chen MX, Cao ZY, Jiang Y, Zhu ZW (2013) Direct determination of glyphosate and its major metabolite, aminomethylphosphonic acid, in fruits and vegetables by mixed-mode hydrophilic interaction/weak anion-exchange liquid chromatography coupled with electrospray tandem mass spectrometry. $\mathrm{J}$ Chromatogr A 1272: 90-99.

5. Goscinny S, Unterluggauer H, Aldrian J, Hanot V, Masselter S (2012) Determination of Glyphosate and Its Metabolite AMPA (Aminomethylphosphonic Acid) in Cereals After Derivatization by Isotope Dilution and UPLC-MS/MS Food Anal Methods 5: 1177-1185.

6. Yoshioka N, Asano M, Kuse A, Mitsuhashi T, Nagasaki Y, et al. (2011) Rapid determination of glyphosate, glufosinate, bialaphos, and their major metabolites in serum by liquid chromatography-tandem mass spectrometry using hydrophilic interaction chromatography. J Chromatogr A 1218: 36753680 .

7. Pline WA, Wilcut JW, Duke SO, Edmisten KL, Wells R (2002) Tolerance and accumulation of shikimic acid in response to glyphosate applications in glyphosate-resistant and nonglyphosate-resistant cotton (Gossypiumhirsutum L.). J Agric Food Chem 50: 506-512. 
Citation: Raji M, Sharkhuu A, Amad M, Merzaban J, Gehring C (2014) Determination of Inter-leaf Translocated Free Glyphosate in Arabidopsis thaliana using Liquid Chromatography Tandem Mass Spectrometry (LC-MS/MS) after Derivatization with Fluorenylmethyloxycarbonyl Chloride (FMOC-CI). J Anal Bioanal Tech S2: 007. doi:10.4172/2155-9872.S2-007

Page 6 of 6

8. Khrolenko MV, Wieczorek PP (2005) Determination of glyphosate and its metabolite aminomethylphosphonic acid in fruit juices using supportedliquid membrane preconcentration method with high-performance liquid chromatography and UV detection after derivatization with p-toluenesulphony chloride. J Chromatogr A 1093: 111-117.

9. Moye H, Miles C, Scherer S (1983) A simplified high-performance liquid chromatographic residue procedure for the determination of glyphosate herbicide and (aminomethyl)phosphonic acid in fruits and vegetables employing postcolumnfluorogenic labeling. J Agric Food Chem 31: 69-72.

10. Ibáñez M, Pozo OJ, Sancho JV, López FJ, Hernández F (2005) Residue determination of glyphosate, glufosinate and aminomethylphosphonic acid in water and soil samples by liquid chromatography coupled to electrospray tandem mass spectrometry. J Chromatogr A 1081: 145-155.

11. Li B, Deng X, Guo D, Jin S (2007) Determination of glyphosate and aminomethylphosphonic acid residues in foods using high performance liquid chromatography-mass spectrometry/mass spectrometry. Se Pu 25: 486-490.

12. Zhu Y, Zhang F, Tong C, Liu W (1999) Determination of glyphosate by ion chromatography. J Chromatogr A 850: 297-301.

13. Bauer KH, Knepper TP, Maes A, Schatz V, Voihsel M (1999) Analysis of polar organic micropollutants in water with ion chromatography-electrospray mass spectrometry. J Chromatogr A 837: 117-128.

14. Stalikas CD, Pilidis GA (2000) Development of a method for the simultaneous determination of phosphoric and amino acid group containing pesticides by gas chromatography with mass-selective detection optimization of the derivatization procedure using an experimental design approach. J Chromatogr A 872: 215225.

Citation: Raji M, Sharkhuu A, Amad M, Merzaban J, Gehring C (2014) Determination of Inter-leaf Translocated Free Glyphosate in Arabidopsis thaliana using Liquid Chromatography Tandem Mass Spectrometry (LC-MS/MS) after Derivatization with Fluorenylmethyloxycarbonyl Chloride (FMOC-Cl). J Anal Bioanal Tech S2: 007. doi:10.4172/2155-9872.S2-007
15. Börjesson E, Torstensson L (2000) New methods for determination of glyphosate and (aminomethyl)phosphonic acid in water and soil. J Chromatogr A 886: 207-216.

16. Tseng SH, Lo YW, Chang PC, Chou SS, Chang HM (2004) Simultaneous quantification of glyphosate, glufosinate, and their major metabolites in rice and soybean sprouts by gas chromatography with pulsed flame photometric detector. J Agric Food Chem 52: 4057-4063.

17. Sancho JV, Hernández F, López FJ, Hogendoorn EA, Dijkman E (1996) Rapid determination of glufosinate, glyphosate and aminomethylphosphonic acid in environmental water samples using precolumnfluorogenic labeling and coupled-column liquid chromatography. J Chromatogr A 737: 75-83.

18. Hetherington PR, Reynolds TL, Marshall G, Kirkwood RC (1999) The absorption, translocation and distribution of the herbicide glyphosate in maize expressing the CP-4 transgene. J Exp Bot 50: 1567-1576.

19. Belles D, Shaner D, Westra P, Brunk G (2006) Comparison of efficacy, absorption and translocation of three glyphosate formulations on velvetleaf (Abutilon theophrasti). Pest Manag Sci 62: 1177-1181.

20. Steinrücken HC, Amrhein N (1980) The herbicide glyphosate is a poten inhibitor of 5-enolpyruvyl-shikimic acid-3-phosphate synthase. Biochem Biophys Res Commun 94: 1207-1212.

21. Ream JE, Yuen HK, Frazier RB, Sikorski JA (1992) EPSP synthase: binding studies using isothermal titration microcalorimetry and equilibrium dialysis and their implications for ligand recognition and kinetic mechanism. Biochemistry 31: 5528-5534.

Submit your next manuscript and get advantages of OMICS Group submissions

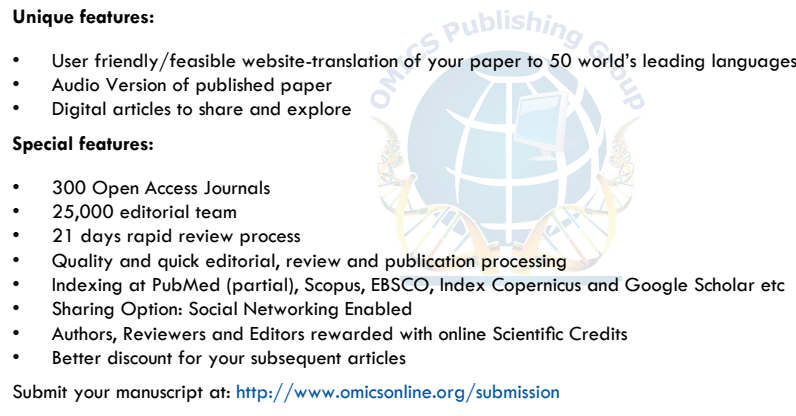

